\title{
SYNOVIAL HYPERTROPHY DETECTED USING ULTRASONOGRAM IN PRIMARY OSTEOARTHRITIC KNEES: PREVALENCE AND CORRELATION WITH RADIOGRAPHIC STAGING
}

\author{
Chawanvuth Termtanun, Thanainit Chotanaphuti, Saradech Khuangsirikul, Danai Heebtamai
}

Department of Orthopedics, Phramongkutklao Hospital, Bangkok, Thailand

\begin{abstract}
Background: Treatment of primary osteoarthritic (OA) knee has changed in recent decades with a greater focus on synovitis as one cause of cartilage destruction and degeneration. Ultrasonography (US), a noninvasive, low cost and convenient procedure may be used for early detection and monitoring synovitis in primary OA knee. Somehow, the lack of data on the prevalence of synovial hypertrophy $(\mathrm{SH})$ and its correlation to disease progression has precluded the use of US in clinical practice.

Objective: The study aimed to determine the prevalence of SH at each stage of the disease and its correlation to structural damage.

Methods: In all, 214 knees among 127 cases diagnosed as having primary OA knee were examined using US. The midline scanning technique of US was performed and synovial thickness at the suprapatellar pouch was observed. All knees were categorized according to the Kellgren-Lawrence radiographic staging (KL). The prevalence of SH in each KL with 2, 2.5 and $3 \mathrm{~mm}$ cutoff level were calculated. The correlation between synovial thickness and KL was also analyzed.

Results: The prevalence of SH with 2 mm cutoff level in KL I-IV was 38.8, 70.8, 66.6 and 91.1\%, respectively. The prevalence of SH with $2.5 \mathrm{~mm}$ cutoff level in KL I-IV was 5.5, 37.5, 35.4 and 74.2\%, respectively. The prevalence of SH with $3 \mathrm{~mm}$ cutoff level in KL I-IV was 0, 29.1, 20.8 and 56.4\%, respectively. The overall prevalence with $2,2.5$ and $3 \mathrm{~mm}$ cut-off level was 72.2, 50.9 and 37.1\%, respectively. Synovial thickness, measured in millimeters, correlated well with $\mathrm{KL}(p<0.01)$. The correlation of synovial thickness between each KL was also statistically significant $(p<0.05)$ except those between KL II and KL III ( $p=0.98)$.

Conclusion: Synovial thickness at the suprapatellar pouch detected with midline scanning US reflected the degree of synovitis which correlated well with structural damage and could be used to monitor disease progression in primary OA knee.
\end{abstract}

Keywords: Synovial hypertrophy, Synovitis, Osteoarthritic knee, Radiographic staging, Ultrasonogram

J Southeast Asian Med Res 2020; 4(1):33-40

http://www.jseamed.org

\section{Correspondence to:}

Termtanun C., Department of Orthopedics, Phramongkutklao Hospital, Bangkok, Thailand Email: c_men@outlook.com

Received: 31 October 2019

Revised: 29 January 2020

Accepted: 14 February 2020 


\section{Introduction}

For several decades, mainstay treatments of primary osteoarthritic (OA) knee were supportive until the end stage of disease in which the joint replacement was inevitable. As the disease progresses, the knee structure decays and the symptoms worsen. ${ }^{(1)}$ Recently, the paradigm of conservative treatment has changed to prevent or delay disease progression by controlling synovitis of the joint. ${ }^{(2-13)}$ However, the imaging modality to monitor the effectiveness of each treatment has not yet been agreed upon. Plain radiography is reflects only irreversible structural damage; thus, cannot be used for early detection. Magnetic resonance imaging study (MRI) is not a cost-effective choice to monitor disease progression in clinical settings. On the other hand, ultrasonogram (US), a noninvasive, low cost and convenient procedure can offer the benefit of early detection and monitor synovial hypertrophy $(\mathrm{SH})$ with comparable accuracy to MRI. ${ }^{(14)}$ Many studies conducted in the US in osteoarthritic knees reported a wide range of $\mathrm{SH}$ prevalences (14.5 to 99.7\%), different cut-off levels of $\mathrm{SH}$ and different correlations with either radiographic staging or symptoms. ${ }^{(15-19)}$ The study aimed to determine the prevalence of $\mathrm{SH}$ at each stage of disease and its correlation to structural damage.

US has been widely used in monitoring synovitis of inflammatory joint disease, usually performed by rheumatologists and radiologists. ${ }^{(15-20)}$ Unfortunately, the archaic concept of pathophysiology in primary osteoarthritic knee, i.e., mechanical wear and tear, has long blinded orthopedic surgeons from the true culprit of cartilage destruction, i.e., subclinical synovitis; thus, the use of US in orthopedic practice is underrated..$^{(4-13)}$

Another concern about US is the variety of techniques and operator dependency, rendering the reliability lower than it should be. The reliability of US in detecting structural abnormalities is low; however, when focusing on only inflammatory abnormalities, the agreement is high despite the experience of the sonographer. ${ }^{(21)}$ Our secondary goal is to determine the reliability of a midline scanning US technique to detect $\mathrm{SH}$ at the suprapatellar pouch.

\section{Methods}

Study population

Patients, visiting the Outpatient Department, Orthopedics Division, Phramongkutklao Hospital and presenting the chief complaint of knee pain, were enrolled in this study from June 2018 to December 2018. This study was approved by the Research Ethics Committee of Phramongkutklao College of Medicine. All participants provided written informed consent.

The inclusion criteria comprised patients receiving a diagnosis of osteoarthritis on either side of the knees according to the ACR clinical classification criteria for knee osteoarthritis and having at least three of the following six items: age $>50$ years, morning stiffness $<30$ minutes, crepitus on knee motion, bony tenderness, bony enlargement and no palpable warmth. ${ }^{(22)}$ The exclusion criteria comprised patients having a historycontributingtosecondaryosteoarthritis,i.e., posttraumatic, postinfection, postsurgery, inflammatory joint disease, vasculitis or any connective tissue disorder.

\section{Ultrasound Instrument}

The GE Healthcare model LOGIQ ${ }^{\circledR}$ e, Preset: Musculoskeletal - knee in B-mode, 12L-RS Wide Band Linear Probe (12MHz) was used in this study.

\section{Technique}

The patient was placed in a supine position on the examination table, keeping his/her knee flexed but relaxed at $30^{\circ}$, one at a time. Midline scanning technique US was performed with a linear probe vertically applied at just proximal to the superior pole of the patella. ${ }^{(23,24)}$ The quadriceps were identified as a parallel line of muscle fibers originating from the superior pole of the patella and were tracked along the quadricep fiber proximally, but not farther than 1 to 2 finger breadth, in the supra-patella pouch. The suprapatellar prefemoral fat pad is just proximal to the anterior part of the femoral condyles. The heterogenous fatty streak confirms the fat pad and is used to differentiate from the synovium, the homogenous echoic layer of tissue overlying the fat pad. When a substantial amount of synovial effusion is encountered, the probe is compressed as much as possible to minimize effusion at the area of interest. ${ }^{18,23,24)}$ The thickest part of the synovium was measured in millimeters to one decimal. The measurements were repeated three times and the thickest value of the synovium was recorded. 

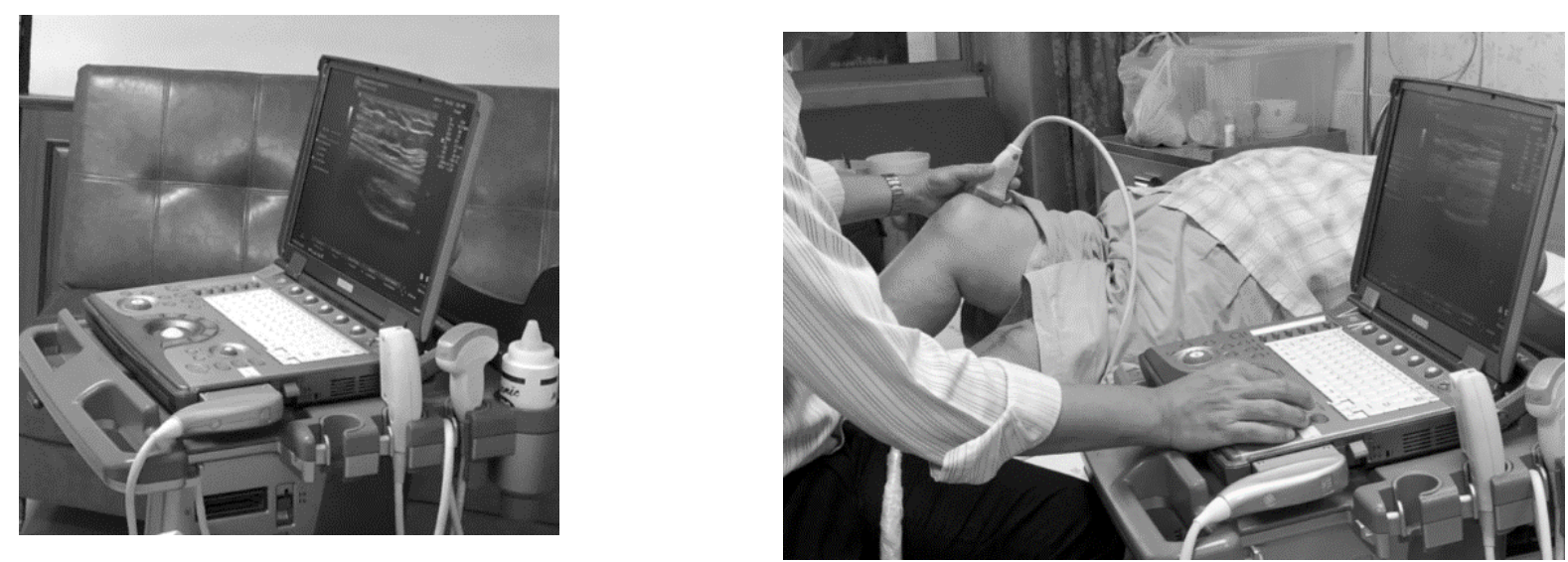

Image 1. Ultrasound machine, midline scanning technique with linear probe
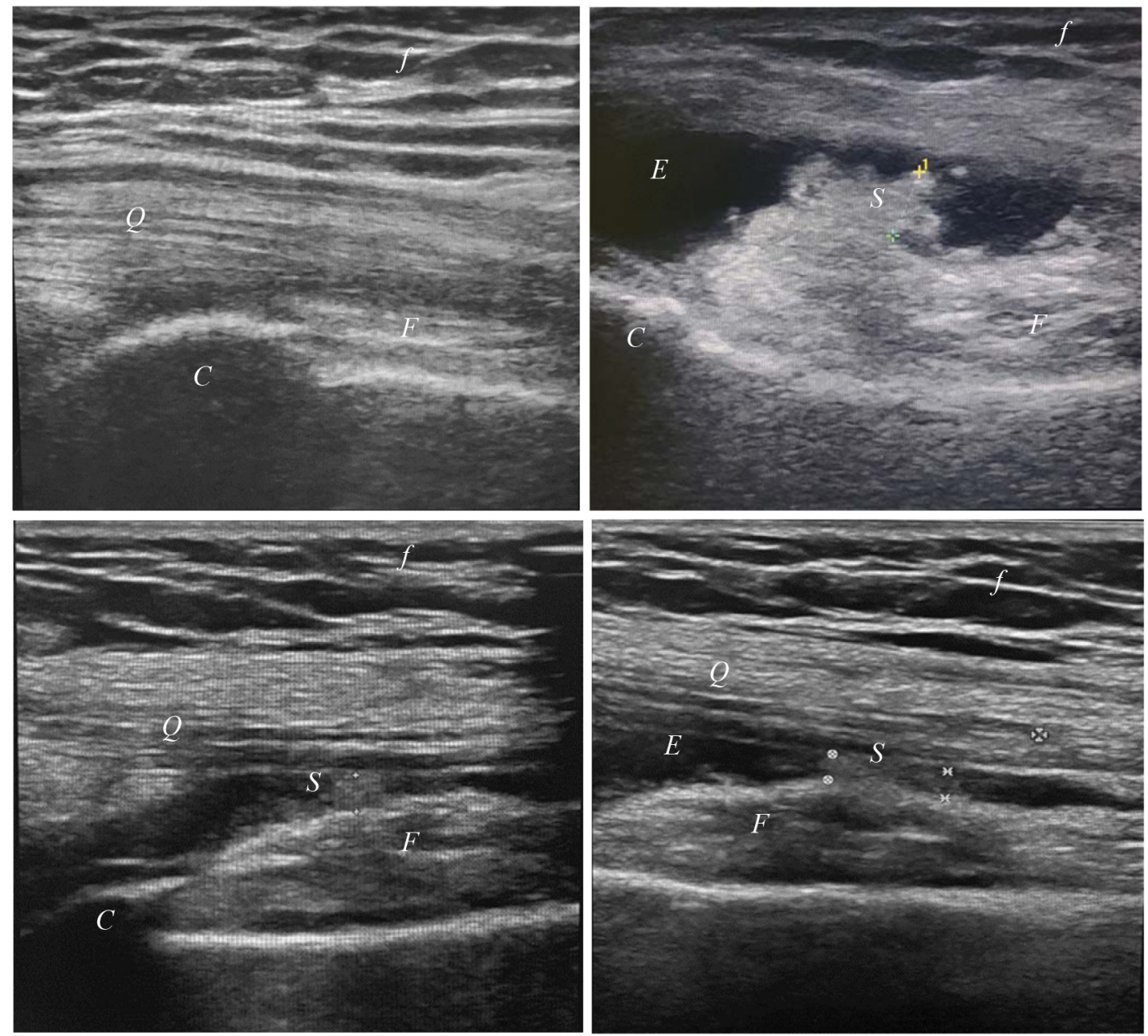

Image 2. Imaging and measuring

(f: subcutaneous fat, Q: quadriceps, F: prefemoral fat pad, S: synovium, C: femoral condyle, E: effusion)

\section{Reliability of measuring synovial thickness}

Under the same midline scanning technique, two sonographers, blinded to patients' history, physical examination and radiographic results, independently performed ultrasonogram in the same $10 \mathrm{OA}$ knees. Intra and interobserver reliability were calculated using intraclass correlation coefficients (ICC) using the Shrout and Fleiss model (1979)

$$
\frac{M S_{R}-M S_{W}}{M S_{R}+(k+1) M S_{W}}
$$


Intra-observer reliability under the model of one-way random effects, absolute agreement and single rater $[\operatorname{ICC}(1,1)]$ were calculated using the formula below.

$$
\frac{M S_{R}-M S_{W}}{M S_{R}}
$$

Interobserver reliability under the model of one-way random effects, absolute agreement, multiple raters $[\operatorname{ICC}(1, \mathrm{k})]$ were calculated using the formula below.

$M S_{R}=$ mean square for rows, $M S_{W}=$ mean square for residual sources of variance, $k=$ number of raters

\section{Radiographic staging}

The standing AP/lateral radiogram of knees was performed and classified under the Kellgren and Lawrence grading system (KL). ${ }^{(25)}$

Because the difference between KL grading of 0 and 1 is subtle and difficult to categorize, they were combined as KL grade $0-1$.

\section{blinding outcome assessors}

Patients' history taking and physical examination were performed by an orthopedic surgeon in the screening room. When the patients' condition met the criteria, after being informed and agreeing to enroll in the study, they were sent to the US room, without any documents describing their history or physical examination. They were sent for radiogram only after their sonogram was completed. In this manner, the sonographers were blinded to the patients' history and radiographic results.

\section{Statistical analysis}

Prevalence was calculated with respect to different cutoff levels of SH at 2, 2.5 and $3 \mathrm{~mm}$ thickness. The correlation between synovial thickness (in $\mathrm{mm}$ ) and $\mathrm{KL}$ was analyzed using One-way ANOVA and Scheffe post-hoc test. The prevalence of $\mathrm{SH}$ in each KL with 2, 2.5 and 3 cutoff levels was calculated. Synovial thickness more than $2 \mathrm{~mm}$ was identified as $\mathrm{SH}$ and calculated with Chi-square test to determine the relationship between $\mathrm{SH}$ and KL.

\section{Results}

Demographic data of enrolled participants Age and sex of the patients in each KL grading were recorded as baseline characteristics as shown in Table 2.

\section{Prevalence of synovial hypertrophy (SH)}

The overall prevalence of SH with $2 \mathrm{~mm}$ cutoff was $72.2 \%$ and the prevalence among KL 0 to 4 were $38.8,70.8,66.6$ and $91.1 \%$, respectively. Using the 2.5 and $3 \mathrm{~mm}$ cutoff values, the prevalences were lower than those of the $2 \mathrm{~mm}$ (as shown in Table 3).

Table 1. Kellgren and Lawrence grading system (KL)

Kellgren and Lawrence grading system (KL)

Grade 0: No feature of osteoarthritis

Grade 1: Doubtful narrowing of joint space and possible osteophytic lipping

Grade 2: Definite osteophytes and possible narrowing of joint space

Grade 3: Moderate multiple osteophytes, definite narrowing of joint space, and some sclerosis and possible deformity of bone ends

Grade 4: Large osteophytes, marked narrowing of joint space, severe sclerosis and definite deformity of bone ends 
Table 2. Demographic data of enrolled participants

\begin{tabular}{cccccc}
\hline & KL0-1 & KL2 & KL3 & KL4 & Total \\
\hline Male & 2 & 10 & 14 & 33 & 59 \\
Female & 15 & 14 & 34 & 92 & 155 \\
Age & $50-80$ & $52-83$ & $50-83$ & $50-86$ & $50-81$ \\
(mean) & $(56)$ & $(65.8)$ & $(67.8)$ & $(71.3)$ & $(68.7)$ \\
Total & 17 & 24 & 48 & 125 & 214 \\
\hline \multicolumn{5}{|}{} \\
\hline
\end{tabular}

Table 3. Prevalence of SH using different cutoff levels

\begin{tabular}{cccccc}
\hline cut-off level & KL 0-1 & KL 2 & KL 3 & KL 4 & Overall \\
\hline $2 \mathrm{~mm}$ & $38.8 \%$ & $70.8 \%$ & $66.6 \%$ & $91.1 \%$ & $72.2 \%$ \\
$2.5 \mathrm{~mm}$ & $5.5 \%$ & $37.5 \%$ & $35.4 \%$ & $74.2 \%$ & $50.9 \%$ \\
$3 \mathrm{~mm}$ & $0 \%$ & $29.1 \%$ & $20.8 \%$ & $56.4 \%$ & $37.1 \%$ \\
\hline
\end{tabular}

\section{Correlation}

Synovial thickness, measured in millimeters, was higher with more advance KL grading. Under one-way ANOVAanalysis, synovial thickness was well-correlated with KL grading $(p<0.01)$. Under the Scheffe posthoc test, the correlation of synovial thickness between each KL was also significant $(p<0.05)$ except those between KL2 and KL3 $(p=0.98)$.

Synovialhypertrophy( $\mathrm{SH})$,i.e., synovial thickness of more than $2 \mathrm{~mm}$, is well-correlated with KL grading under the Chi-square test $(p<0.01)$ These significances were also found at 2.5 and $3 \mathrm{~mm}$ cut-off levels.

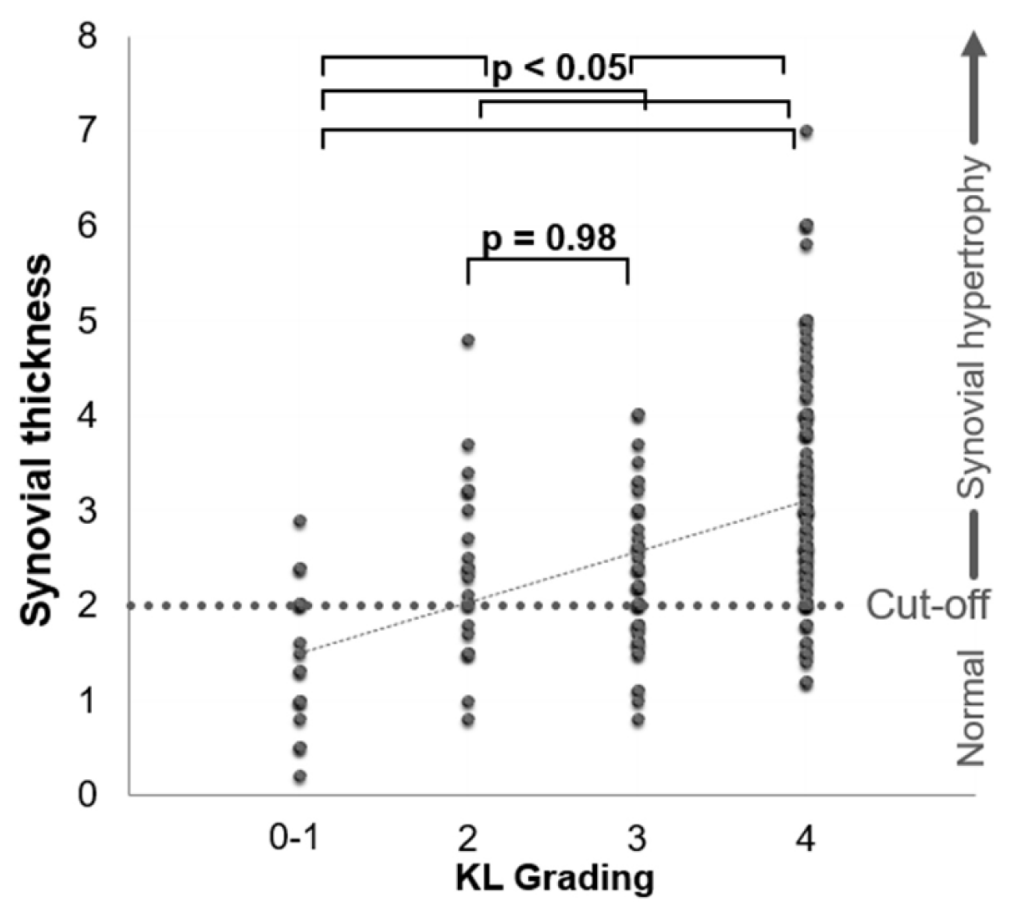

Figure 1. Correlation between synovial thickness and KL grading under one-way ANOVA 
Table 4. Repeated measurement of synovial thickness under midline scanning technique performed by two independent sonographers.

\begin{tabular}{|c|c|c|c|c|c|c|c|c|c|}
\hline \multirow[b]{3}{*}{ Knee } & & \multicolumn{4}{|c|}{ Rater1 } & \multicolumn{4}{|c|}{ Rater2 } \\
\hline & & 2nd & & & Final & 2nd & & & Final \\
\hline & 1 & 2.5 & 2.8 & 1.8 & 2.8 & 2 & 1.8 & 3.5 & 3.5 \\
\hline Knee & 2 & 3.1 & 2.5 & 3.5 & 3.5 & 2.2 & 3 & 2.6 & 3 \\
\hline Knee & 3 & 3 & 2.6 & 3 & 3 & 2.4 & 2 & 1.8 & 2.4 \\
\hline Knee & 4 & 2.6 & 1.9 & 2 & 2.6 & 3 & 3.3 & 3.1 & 3.3 \\
\hline Knee & 5 & 0.9 & 1.8 & 1.4 & 1.8 & 2.5 & 1.6 & 1 & 2.5 \\
\hline Knee & 6 & 2.6 & 2.4 & 2 & 2.6 & 2.2 & 3.2 & 2.6 & 3.2 \\
\hline Knee & 7 & 1.6 & 2 & 2.4 & 2.4 & 2.3 & 1.9 & 2.8 & 2.8 \\
\hline Knee & 8 & 1.5 & 1.4 & 2 & 2 & 1 & 0.4 & 0.8 & 1 \\
\hline Knee & 9 & 4.2 & 3.6 & 4.2 & 4.2 & 2.7 & 3.6 & 3.1 & 3.6 \\
\hline Knee & 10 & 3 & 3.8 & 3.6 & 3.8 & 2.8 & 2.2 & 3.4 & 3.4 \\
\hline
\end{tabular}

\section{Inter- and Intra-observer reliability}

After a period of practicing the same protocol of midline scanning technique of two sonographers, 10 random osteoarthritic knees were examined and repeated three times use of US in each knee and sonographers. The highest value of synovial thickness was concluded as the final value.

For Rater1, intra-observer reliability was good to excellent (ICC's: $0.79(0.53-0.93)$ to 0.92 (0.77-0.98). For Rater2, intra-observer reliability was moderate to good (ICC's: $0.60(0.22-0.86)$ to $0.82(0.46-0.95)$.

Synovial thickness was observed with moderate to good inter-observer reliability (ICC's: $0.64(0.02-0.89)$ to $0.78(0.04-0.94)$. During this study, in terms of our experience, Rater1 had already performed sonogram under midline scanning technique over 200 cases compared with only 30 cases performed by Rater 2 .

\section{Discussion}

Synovial thickness at the supra-patellar pouch, measured either in $\mathrm{mm}$ or percentage of $\mathrm{SH}$, is well-correlated with radiographic staging. In advanced stage of osteoarthritic knees with severe structural damage, the synovium are thicker than early stages. These findings suggested that long standing synovitis in the joint may thicken the synovium over time. Thin synovium can be found in KL4 and may be the result of shrinkage under fibrotic processes. Further study is needed to prove this assumption.

The prevalence of $\mathrm{SH}$ varied with different cut-off levels; however, the $2 \mathrm{~mm}$ cut-off level was more sensitive in early stages of osteoarthritic knees with prevalence $38.8 \%$ in KL0 to 1 . On the other hand, the synovial thickness of less than $2 \mathrm{~mm}$ should be considered normal, to prevent overdiagnosis of SH with US.

In this study, the overall prevalence among each cut-off levels ( 37.1 to $72.2 \%$ ) were within the range of related studies $(22.1 \text { to } 82.5 \%)^{(15-19)}$

The correlation of $\mathrm{SH}$ to structural damage was similar to a majority of related studies ${ }^{(15-19)}$ but distinct from some studies. ${ }^{(17,18)}$ The different methods of collecting data may limit the comparison of result across studies.

The concern about operator dependency of US has long hindered a majority of orthopedics in trying or practicing US. Better than expectation, we found that the midline scanning technique for US was easy to perform with acceptable reliability and was easy to improve, even among inexperienced practitioners.

\section{Conclusion}

Plain radiograms could represent irreversible structural damage and are conventionally used as tools to classify stages of osteoarthritis. In this study, the more advanced the stage of osteoarthritic knees became, the higher prevalence of SH was observed. The trend of synovial thickness also increased at each stage; thus, correlating with structural damage. These findings suggest that long standing synovitis contributed to cartilage destruction. Similar to secondary osteoarthritis from inflammatory disease, primary osteoarthritis also presents inflammation, even to a much lesser degree, but prolonged enough to 
thicken the synovium to a detectable range in US. Conservative treatment of primary osteoarthritic knee should aim to prevent structural damage of the cartilage by controlling the synovitis process. Treatment should not be halted until the synovitis is well-controlled, even when the symptoms are minimized by medication or activity reduction because the fluctuating pain did not correlate with the tedious progression of osteoarthritis, especially during early stage osteoarthritis, when structural damage has yet to occur. Salvaging the cartilage during early stage osteoarthritic knees is paramount.

Unfortunately, subclinical synovitis is subtle concerning physical examination alone, presently, and US may be the most practical method of monitoring synovitis. With any mode of treatment applied, US will also detect its effectiveness, rather than subjective pain. This study brings a new paradigm one step closer regarding osteoarthritic treatment - to delay or even prevent osteoarthritis against the sands of time.

\section{References}

1. Wang Y, Teichtahl AJ, Abram F, Hussain SM, Pelletier JP, Cicuttini FM, Martel-Pelletier J. Knee pain as a predictor of structural progression over 4 years: data from the Osteoarthritis Initiative, a prospective cohort study. Arthritis Res Ther 2018; 20: 250.

2. Mathiessen A, Conaghan PG, Synovitis in osteoarthritis: current understanding with therapeutic implications. Arthritis Res Ther 2017; 19: 18.

3. Felson DT, Niu J, Neogi T, Goggins J, Nevitt $\mathrm{MC}$, Roemer $\mathrm{F}$ et al. Synovitis and the risk of knee osteoarthritis: the most study. Osteoarthritis Cartilage 2016; 24: 458-64.

4. Chu CR, Millis MB, Olson SA. Osteoarthritis: From palliation to prevention: AOA critical issues. J Bone Joint Surg Am 2014; 96:e130.

5. Heidari B. Knee osteoarthritis prevalence, risk factors, pathogenesis and features: Part I. Caspian J Intern Med 2011; 2:205-12.

6. Roos EM, Arden NK. Strategies for the prevention of knee osteoarthritis. Nat Rev Rheumatol 2016; 12: 92-101.

7. Berenbaum F. Osteoarthritis as an inflammatory disease (osteoarthritis is not osteoarthrosis!). Osteoarthr Cartil 2013; 21:16-21.
8. Daghestani HN, Kraus VB. Inflammatory biomarkers in osteoarthritis. Osteoarthritis Cartil 2015; 23: 1890-6.

9. Malfait AM. Osteoarthritis year in review 2015: Biology. Osteoarthr Cartil 2016 ; 24: 21-6.

10. Orlowsky EW, Kraus VB. The role of innate immunity in osteoarthritis: When our first line of defense goes on the offensive. J Rheumatol 2015; 42: 363-71.

11. Scanzello CR, Goldring SR. The role of synovitis in osteoarthritis pathogenesis. Bone 2012; 51: 249- 57.

12. Sellam J, Berenbaum F. Is osteoarthritis a metabolic disease? Jt Bone Spine 2013; 80: 568-73.

13. Varady NH, Grodzinsky AJ. Osteoarthritis year in review 2015: Mechanics. Osteoarthr Cartil 2016; 24: 27-35.

14. Iagnocco A. Imaging the joint in osteoarthritis: a place for ultrasound? Best Pract Res Clin Rheumatol 2010; 24: 27-38.

15. Sarmanova A, Hall M, Moses J, Doherty M, Zhang W. Synovial changes detected by ultrasound in people with knee osteoarthritis a meta-analysis of observational studies. Osteoarthr Cartil 2016; 24: 1376-83.

16. D'Agostino MA, Conaghan P, Le Bars M, Baron G, Grassi W, Martin-Mola E, et al. EULAR report on the use of ultrasonography in painful knee osteoarthritis. Part 1: prevalence of inflammation in osteoarthritis. Ann Rheum Dis 2005; 64: 1703-9.

17. Mermerci BB, Garip Y, Uysal RS, Dogruel $\mathrm{H}$, Karabulut E, Ozoran K, et al. Clinic and ultrasound findings related to pain in patients with knee osteoarthritis. Clin Rheumatol 2011; 30: 1055-62.

18. Iagnocco A, Meenagh G, Riente L, Filippucci E, Delle Sedie A, Scire CA, et al. Ultrasound imaging for the rheumatologist XXIX. Sonographic assessment of the knee in patients with osteoarthritis. Clin Exp Rheumatol 2010; 28: 643-6.

19. Hall M, Doherty S, Courtney P, Latief K, Zhang W, Doherty M. Synovial pathology detected on ultrasound correlates with the severity of radiographic knee osteoarthritis more than with symptoms. Osteoarthr Cartil 2014; 22: 1627e33 
20. Karim Z, Wakefield RJ, Quinn M, Conaghan PG, Brown AK, Veale DJ, et al. Validation and reproducibility of ultrasonography in the detection of synovitis in the knee: a comparison with arthroscopy and clinical examination. Arthritis Rheum 2004; 50: 387-94.

21. Iagnocco A, Perricone C, Scirocco C, Ceccarelli F, Mariagrazia M, Gattamelata A, et al. The interobserver reliability of ultrasound in knee osteoarthritis. Rheumatology (Oxford). 2012; 51: 2013-9.

22. Altman R, Asch E, Bloch D. Development of criteria for the classification and reporting of osteoarthritis: Classification of osteoarthritis of the knee. Arthritis Rheum 1986; 29: 1039-49. 23. Alves TI, Girish G, Kalume Brigido M, Jacobson JA, US of the Knee: Scanning Techniques, Pitfalls, and Pathologic Conditions. Radiographics. 2016 ;36: 1759-1775.

24. Gandikota G, Tun M. Ultrasound of the hip in rheumatology. Indian J Rheumatol 2018; 13: 29-35.

25. Kohn MD, Sassoon AA, Fernando ND. Classifications in Brief: Kellgren-Lawrence Classification of Osteoarthritis. Clin Orthop Relat Res 2016 ; 474: 1886-93. 